\title{
U.S. HOSPITAL CHARGES IN MASTECTOMY: EMPIRICAL ANALYSES USING PATIENT, HOSPITAL AND TREATMENT VARIABLES
}

Anne Fiedler, Nova Southeastern University. U.S.A.

Ravi Chinta, Nova Southeastern University. U.S.A.

Ramdas Chandra, Nova Southeastern University. U.S.A.

dx.doi.org/10.18374/JIFE-20-1.2

\begin{abstract}
Breast cancer and, more specifically, related mastectomies, are putting a strain on the healthcare system. The objective of this study is to identify statistically significant contributors of hospital charges for mastectomy hospitalizations, and empirically suggest cost reduction reforms in mastectomy treatments and patient care. The AHRQ's (Agency for Healthcare Research and Quality) is a federal agency data that gathers data on in-patient hospitalization episodes for all Diagnosis Related Groups (DRGs) used to classify all disease conditions for which patients are admitted to hospitals. This research used the AHRQ's data on Mastectomy treatments (DRG codes 582 and 583). Employing regression analyses on a total of 1921 cases, we analyzed hospital charges as a dependent variable that is a function of patient \& hospital characteristics, and the specific patient care rendered. Our results show that the most important determinants of hospital charges were length of stay and control-ownership of the hospital. The number of procedures, severity of illness, median household income, primary expected payer, location/teaching status of the hospital, and risk of mortality were also significant. These results identify statistically significant factors that determine hospital charges. Such evidence-based results lead directly to focused cost reforms that can reduce hospitalization charges for mastectomies. While our study is focused on mastectomies replicating our research for other DRGs is is sine-qua-non for cost reforms in the healthcare sector and in-hospital care in particular.
\end{abstract}

Keywords: Hospital Charges; Mastectomy; DRG 582; DRG 583; Patient Demographics; Hospital Control, Payer 